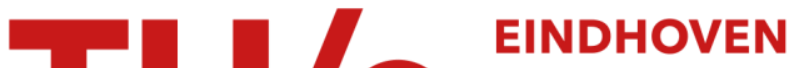 \\ UNIVERSITY OF \\ TECHNOLOGY
}

\section{Simulation of large interconnect structures using ILU-type preconditioner}

\section{Citation for published version (APA):}

Harutyunyan, D., Schoenmaker, W., \& Schilders, W. H. A. (2008). Simulation of large interconnect structures using ILU-type preconditioner. (CASA-report; Vol. 0836). Technische Universiteit Eindhoven.

\section{Document status and date:}

Published: 01/01/2008

\section{Document Version:}

Publisher's PDF, also known as Version of Record (includes final page, issue and volume numbers)

\section{Please check the document version of this publication:}

- A submitted manuscript is the version of the article upon submission and before peer-review. There can be important differences between the submitted version and the official published version of record. People interested in the research are advised to contact the author for the final version of the publication, or visit the $\mathrm{DOI}$ to the publisher's website.

- The final author version and the galley proof are versions of the publication after peer review.

- The final published version features the final layout of the paper including the volume, issue and page numbers.

Link to publication

\section{General rights}

Copyright and moral rights for the publications made accessible in the public portal are retained by the authors and/or other copyright owners and it is a condition of accessing publications that users recognise and abide by the legal requirements associated with these rights.

- Users may download and print one copy of any publication from the public portal for the purpose of private study or research.

- You may not further distribute the material or use it for any profit-making activity or commercial gain

- You may freely distribute the URL identifying the publication in the public portal.

If the publication is distributed under the terms of Article 25fa of the Dutch Copyright Act, indicated by the "Taverne" license above, please follow below link for the End User Agreement:

www.tue.nl/taverne

Take down policy

If you believe that this document breaches copyright please contact us at:

openaccess@tue.nl

providing details and we will investigate your claim. 


\title{
Simulation of large interconnect structures using ILU-type preconditioner
}

\author{
D. Harutyunyan, W. Schoenmaker, and W. H. A. Schilders
}

\begin{abstract}
For a fast simulation of interconnect structures we consider preconditioned iterative solution methods for large complex valued linear systems. In many applications the discretized equations result in ill-conditioned matrices, and efficient preconditioners are indispensable to solve the linear systems accurately. We apply the dual threshold incomplete LU (ILUT) factorization as preconditioners for the BICGSTAB iterative solver. On complicated problems with a different range of frequencies we show that the BICGSTAB method with the ILUT preconditioner provides a very accurate solution of the linear systems.
\end{abstract}

\section{Introduction}

With the increasing complexity of on-chip interconnect structures more robust and fast simulation methods are necessary to understand the behavior of electromagnetic fields in such complex structures. For a better understanding of the performance of these structures field simulation approaches provide more insight about the behavior of the electromagnetic fields.

The governing equations of the electromagnetic fields are given by the Maxwell equations. For many applications the potential formulation of the Maxwell equations is used which has several advantages. In particular, for interconnect structures the potential formulation allows separate modeling of fields in dielectric, semiconductor and metallic regions, which reduces the computational time essentially [1,2].

D. Harutyunyan, W. H. A. Schilders

Technische Universiteit Eindhoven, Den Dolech 2, 5612 AZ Eindhoven, The Netherlands and NXP Semiconductors, High Tech Campus 37, 5656 AE Eindhoven, The Netherlands, e-mail: d.harutyunyan@tue.nl, wil.schilders@nxp.nl

W. Schoenmaker Magwel NV, Martelarenplein 13, B-3000 Leuven, Belgium.

e-mail: wim.schoenmaker@magwel.com 
The differential operators are discretized using the usual finite-volume methods for the electric potential and the charge density. However, for the magnetic vector potential the finite-volume method is replaced by a 'finite-surface method' [3], whose origin is found in Stokes' theorem in contrary to the finite-volume methods that are rooted in Gauss' theorem [1]. This discretization method preserves important physical characteristics of the electromagnetic fields at the discrete level, and we obtain physically relevant solutions. After the discretization of the differential equations we obtain a linear system of equations of the form $A x=b$, where the coefficient matrix $A$ is a large scale, sparse and complex valued. For large scale problems direct linear solvers are not always possible to implement, and Krylov subspace methods are common tools to solve linear systems approximately. The performance of Krylov subspace methods highly depends on the condition number of the matrix, and for complicated real life problems the resulting matrix $A$ is usually ill-conditioned. For such problems Krylov subspace methods either require too many iteration steps for the convergence or, in the worst case, they do not converge at all. To overcome these difficulties a good matrix preconditioner can significantly improve the convergence rate of the Krylov subspace methods.

In this paper we apply the BICGSTAB iterative solution algorithm [4] with the ILUT preconditioner [5]. In two problems we show that the ILUT preconditioner improves the convergence rate of BICGSTAB algorithm significantly, and provides a very accurate solution for the linear system.

\section{Potential formulation of the Maxwell equations}

Mathematical modeling of the electromagnetic fields is given by the Maxwell equations:

$$
\begin{array}{lll}
\partial_{t} \mathbf{D}=\nabla \times \mathbf{H}-\mathbf{J}, & \text { (1a) } & \nabla \cdot \mathbf{D}=\rho, \\
\partial_{t} \mathbf{B}=-\nabla \times \mathbf{E}, & \text { (1b) } & \nabla \cdot \mathbf{B}=0,
\end{array}
$$

where $\mathbf{E}$ and $\mathbf{H}$ ( $\mathbf{D}$ and $\mathbf{B}$ ) are the electric and magnetic fields (respectively, the electric and the magnetic flux densities). The following constitutive relations hold for linear media:

$$
\mathbf{D}=\varepsilon \mathbf{E}, \quad \mathbf{B}=\mu \mathbf{H}, \quad \text { (2) } \quad \mathbf{J}=\sigma \mathbf{E}+\mathbf{J}_{\text {diff }},
$$

where the dielectric permittivity $\varepsilon\left(=\varepsilon_{0} \varepsilon_{r}\right)$, the conductivity $\sigma$, and the magnetic permeability $\mu\left(=\mu_{0} \mu_{r}\right)$ are assumed to be space dependent positive definite tensors. The diffusive part of the carrier flows is denoted by $\mathbf{J}_{\text {diff. }}$. This way of writing the current allows us to deal with metals in which the diffusive currents are negligible, as well as with semiconductors. In the latter case the first term represents the drift terms of the electron and hole currents. The free space dielectric permittivity and magnetic permeability are defined by $\varepsilon_{0}$ and $\mu_{0}$, respectively. Before entering into the manipulations of the Maxwell equations, we will discuss some subtle issues that are present in the above set of equations, and which are quite important for a 
successful numerical solution. The electric field $\mathbf{E}$ is a 1-form in the differential geometrical meaning. It implies that it is an object assigned to a link of a computational grid. The displacement $\mathbf{D}$ is a 2 -form and should be assigned to surface elements of the computational grid. To be more precise, since $\rho$ is a 3-form, which can be shown to be equivalent to a 0 -form in three dimensions, we may assign $\rho$ to the nodes of the grid, and therefore $\mathbf{D}$ can be assigned to the faces of the dual grid. On the other hand, the 2-form $\mathbf{B}$ is assigned to the faces of the primary grid. The 1-form $\mathbf{H}$ is assigned to the links of the dual grid. When keeping these considerations in mind it becomes evident how the Hodge operators, $\varepsilon, \mu$ and $\sigma$ must be handled while setting up the discretization. Moreover, by knowing in which grid and to which object in the grid a variable is assigned, we are able to give meaning to expressions such as $\nabla$. E. Strictly speaking, the $\nabla$. operator should act on a 2form. However, since the normal on a face of the dual grid coincides with a link of the primary grid, we can apply the finite-volume technique to this expression in the primary grid. More details about the discretization methods can be found in $[1,3]$.

We will now proceed with rewriting of the Maxwell equations. From the Maxwell equations (1d) and (1b) it follows that there is a vector potential $\mathbf{A}$ and a scalar potential $V$ such that

$$
\mathbf{B}=\nabla \times \mathbf{A}, \quad \text { (4) } \quad \mathbf{E}=-\partial_{t} \mathbf{A}-\nabla V .
$$

If we substitute (4) and (5) into (1), the potential formulation of the Maxwell equations in the frequency domain can be written as [1]

$$
\begin{aligned}
& \nabla \times \mu^{-1} \nabla \times \mathbf{A}-(\sigma+j \omega \varepsilon)(-j \omega \mathbf{A}-\nabla V)=\mathbf{J}_{\text {diff }}, \\
& \nabla \cdot(\varepsilon(\nabla V+j \omega \mathbf{A}))=-\rho, \quad \text { in insulators and semiconductors, } \\
& \nabla \cdot((\sigma+j \omega \varepsilon)(\nabla V+j \omega \mathbf{A}))=0, \quad \text { in metals. }
\end{aligned}
$$

For completeness, the 1-form $\mathbf{A}$ is assigned to the links of the primary grid and the potential $V$ is assigned to the nodes of the primary grid. For the unique solution of (6) we use the following gauge condition which is linear in the scalar and vector potentials, namely

$$
\frac{1}{\mu_{0}} \nabla(\nabla \cdot \mathbf{A})+j \omega \varepsilon \xi \nabla V=0,
$$

where $0 \leq \xi \leq 1$. The above equation resembles the Coulomb gauge for $\xi=0$ and the Lorentz gauge for $\xi=1$ and constant $\varepsilon$. For the discretization of (6) and (7) we refer to $[1,3]$.

\section{Short review on ILU preconditioners}

There are two class of general purpose preconditioners for large linear systems: ILU preconditioners and sparse approximate inverse preconditioners. Among ILU preconditioners a common approach is to use ILU(0) factorization which uses a fixed 
sparsity pattern. This preconditioner is very ineffective for our problems, and the iterative method does not converge with this preconditioner. An improvement is to use $\operatorname{ILU}(\tau)$ factorization, which allows more fill-in depending on the drop tolerance $\tau$. The drawback of this method is that the memory requirement is unknown in advance. For ill-conditioned problems a small drop tolerance $\tau$ is required to construct a good ILU $(\tau)$ preconditioner, but for large problems the factorization is not possible because of memory limitations. The construction of a sparse approximate inverse for ill-conditioned problems is far more complicated and time consuming.

To overcome the memory limitation problems of an $\operatorname{ILU}(\tau)$ preconditioner when applied to complicated problems we use a dual threshold $\operatorname{ILUT}(p, \tau)$ preconditioner. Similar to the $\operatorname{ILU}(\tau)$ preconditioner, the same dropping rule is applied based on the drop tolerance $\tau$, then only $p$ largest elements in the row of the $\mathrm{L}$ and $\mathrm{U}$ matrices are kept. In the course of factorization $\tau$ controls the computational cost while $p$ controls the computer memory, for details see [5].

The computational time required for the ILUT factorization can be reduced by proper reordering of the matrix elements. There are several reordering algorithms based on different methods. In our experiments we make a comparison between two common reordering methods. The first method is the symmetric reverse CuthillMcKee reordering (SYMRCM) [6] and the second method is the approximate minimum degree (AMD) reordering [7].

\section{Numerical experiments}

In all experiments the iterative procedure is stopped if the 2-norm of the relative residual (relative to the 2-norm of $b$ ) is reduced by a factor $10^{-12}$. We have chosen a small reduction factor in order to observe the validity range of the preconditioner. Several notations are used to show the properties of the preconditioner. The CPU time required to construct the ILUT preconditioner is denoted by Pr-time and the CPU time of BICGSTAB iterations is denoted by It-time. The density ratio of the preconditioned versus the original system is denoted by Ratio $=n n z(L+U) / n n z(A)$.
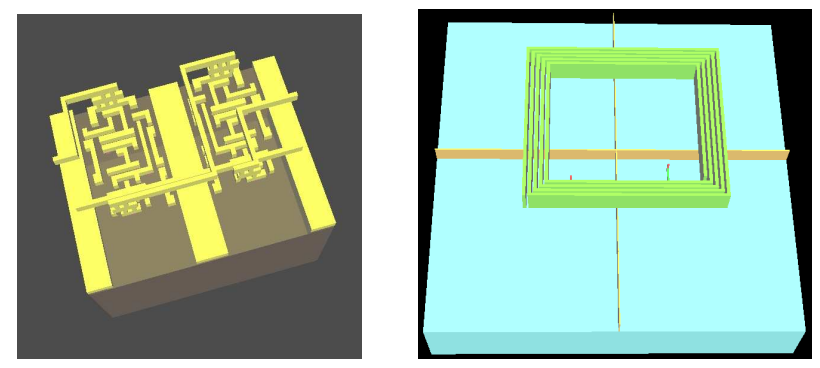

Fig. 1 Left: Interconnect structure of Test case 1. Right: On-chip inductor of Test case 2. 


\subsection{Test case 1}

In this section we present numerical experiments on an interconnect structure, see Fig. 1 (left), with dimension in micrometers $4.4 \times 5.5 \times 4.24$. This test case is provided by NXP semiconductors, where the operating frequency is $500 \mathrm{MHz}$.

First we consider a coarse mesh which results in a matrix of dimension 141513. The convergence diagram of the relative residual is given in Fig. 2. Because the matrix is ill-conditioned it is required to choose a small value for the drop tolerance $\tau$ to achieve convergence. Then a proper choice of $p$ is found depending on the complexity of the matrix and memory limitations. Note that with the chosen values of the drop tolerance it was impossible to construct the $I L U(\tau)$ preconditioner because of memory limitations. With the AMD reordering the iterative method requires significantly less number of iterations as compared to the SYMRCM reordering. More detailed performance information about both methods is given in Table 1. It is clear that the AMD reordering requires less time to construct the preconditioner, and the iteration time with the AMD reordering is much smaller as compared to the iteration time with the SYMRCM reordering. Furthermore, we note that with the AMD reordering the required fill-in of the preconditioner is less than that with the SYMRCM reordering. Because of the space limitation we do not show a similar table for the other experiments, but all of the above observations hold true for all our experiments.

We perform a similar experiment on a fine mesh which results in a matrix of dimension 428710. This case is more difficult and requires larger value of $p$ and smaller drop tolerance $\tau$ to obtain an accurate solution. The convergence diagram of the relative residual with the AMD reordering is given in Fig. 3 (right). Let us mention that we failed to obtain convergence with the SYMRCM reordering.

Distribution of the 25 smallest magnitude eigenvalues of the original matrix and the preconditioned matrix computed by the Jacobi-Davidson method is given in Fig. 3 (left). It is clear that the smallest magnitude eigenvalues of the preconditioned matrix are shifted away from the origin, which explains the good convergence behavior of the BICGSTAB method with the ILUT preconditioner.

Table 1 Test case 1.Performance of the preconditioner with the two different reordering methods.

\begin{tabular}{|c|c|c|c|c|c|c|c|c|c|}
\hline & & \multicolumn{3}{|c|}{ SYMRCM } & \multicolumn{4}{c|}{ AMD } \\
\hline$p$ & $\tau$ & Pr-time & It-time & Its & Ratio & Pr-time & It-time & It & Ratio \\
\hline 100 & $10^{-7}$ & 1029.53 & 325.65 & 430 & 9.39 & 618.13 & 111.80 & 189 & 6.24 \\
\hline 100 & $10^{-8}$ & 1383.33 & 579.21 & 680 & 9.40 & 814.00 & 150.54 & 231 & 6.28 \\
\hline 150 & $10^{-7}$ & 1867.49 & 300.55 & 303 & 13.94 & 1046.66 & 76.25 & 104 & 8.62 \\
\hline 150 & $10^{-8}$ & 2231.82 & 273.94 & 273 & 13.99 & 1154.57 & 74.46 & 99 & 8.71 \\
\hline 200 & $10^{-6}$ & 1807.44 & 600.37 & 472 & 18.21 & 986.45 & 122.82 & 147 & 10.57 \\
\hline
\end{tabular}



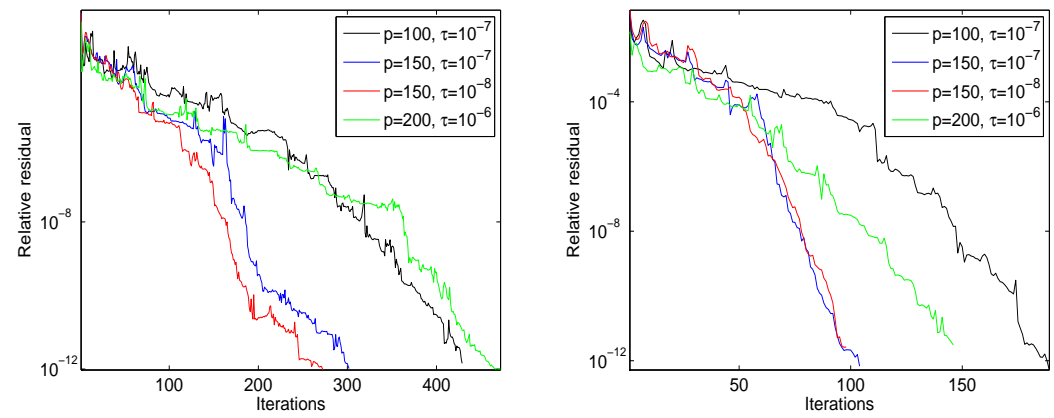

Fig. 2 Test case 1. Convergence diagram of the relative residual on the coarse mesh with the $\operatorname{ILUT}(p, \tau)$ preconditioner. Left: SYMRCM reordering, right: AMD reordering.
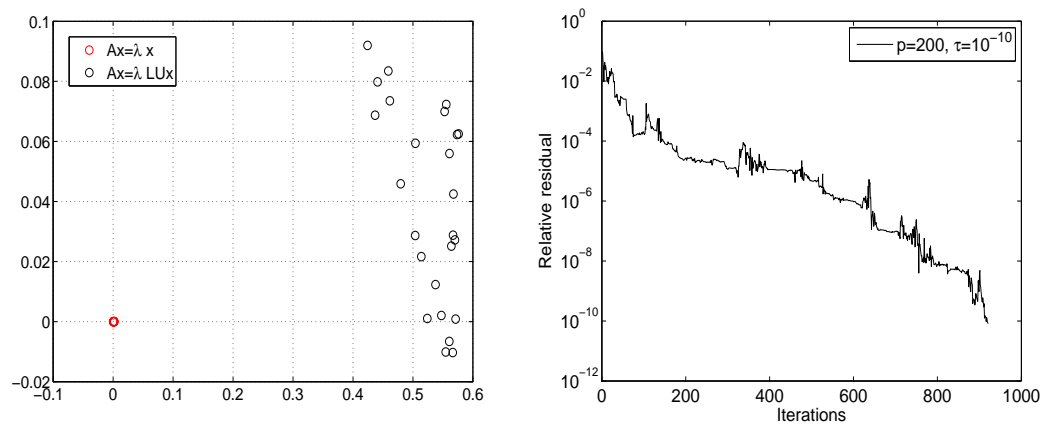

Fig. 3 Test case 1. Left: Distribution of the 25 smallest magnitude eigenvalues on the coarse mesh of the original matrix and the preconditioned matrix with the $\operatorname{ILUT}(p, \tau)$ preconditioner with the AMD reordering. Right: Convergence diagram of the relative residual on the fine mesh with the $\operatorname{ILUT}(p, \tau)$ preconditioner with the AMD reordering.

\subsection{Test case 2}

In the following numerical experiments we consider an on-chip inductor. The dimension of the structure in micrometers is $1000 \times 1000 \times 407$. The inductor with 4.5 windings is provided by austriamicorsystems and contains a pattern of nwell implants below the inductor in the active device layer. This pattern is mimicked here by the large cross in Fig. 1 (right). The goal of this pattern is to reduce eddy currents in the substrate. The inductor is processed in the M4 (the 4th metal layer) and the underpath is found in M3. The goal of the electromagnetic field solving is to compute the quality factor $\mathrm{Q}$, the inductance $\mathrm{L}$ and the resistance $\mathrm{R}$. These variables are extracted from the admittance parameters $Y_{i j}$. The simulation results for the Y-parameters as well as the measurement results are shown in Fig. 4. 

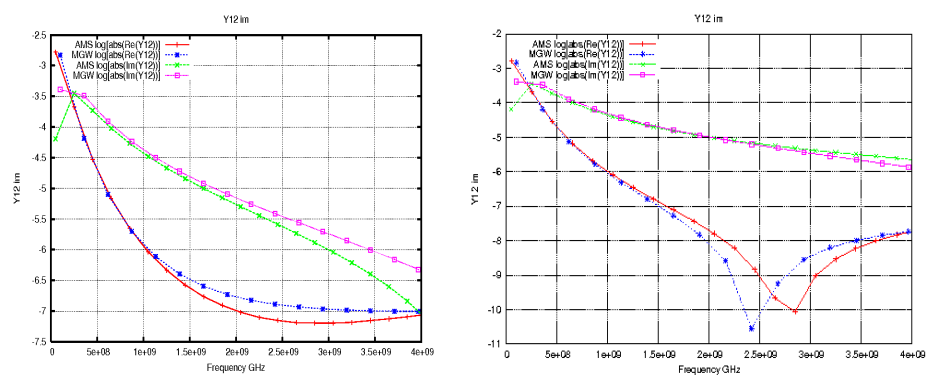

Fig. 4 Test case 2. Comparison of the real and imaginary parts of the Y11 and Y12 parameters.

In our applications for the solution of the linear systems the AMD reordering has proved to be more efficient than the SYMRCM reordering, therefore in the following experiments we use only AMD reordering. Convergence diagrams of the relative residual of the BICGSTAB iterative method with the ILUT preconditioner for the frequencies of $1 \mathrm{GHz}$ and $10 \mathrm{GHz}$ are shown in Fig. 5. As it is expected, for higher frequencies more fill-in, smaller drop tolerance and more iterations are required to achieve the same order of accuracy.

In practice the choice of the parameters $p$ and $\tau$ is more based on the problem and experience, see also [5]. In our applications we have made the following observations:

- For a fixed value of $p$ and $\tau_{0}$, for which a convergence is reached, further decreasing the drop tolerance $\tau<\tau_{0}$ the number of iterations does not decrease significantly but instead it requires much more time for the construction of the preconditioner.

- For a fixed value of $\tau$ (or $p$ ) by increasing the fill-in parameter far enough (or by decreasing the drop tolerance ) the required time for BICGSTAB iterations is almost constant and the most time is spent of the construction of the preconditioner.

Based on our experience we suggest in practical applications for difficult problems to start with $p \approx 50$ and $\tau \approx 10^{-5}$ and then follow how the error of the iterative method behaves. If convergence is not reached then based on the first observation we suggest at first to increase the fill-in parameter. If no convergence is reached then decrease also the drop tolerance.

\section{Conclusions}

We discussed simulation of interconnect structures where the resulting linear systems after space discretization are large and ill-conditioned. We have shown that the ILUT preconditioner is well applicable for these large and difficult problems and 

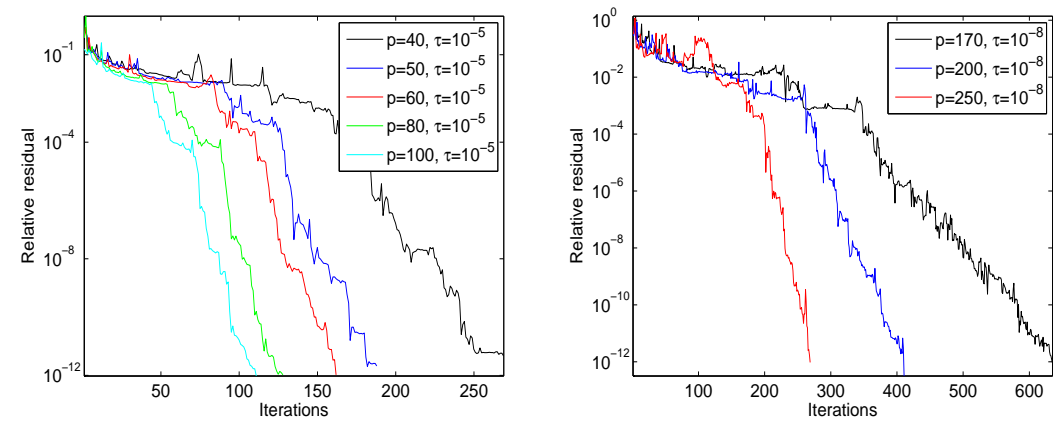

Fig. 5 Test case 2. Convergence diagram of the relative residual with the $\operatorname{ILUT}(p, \tau)$ preconditioner. Left: Frequency is $1 \mathrm{GHz}$, right: Frequency is $10 \mathrm{GHz}$.

provides very accurate solution. The use of AMD reordering is necessary for such complicated problems. The performance of the preconditioner was demonstrated in two different structures.

Acknowledgements The first author is financed by the European Commission through the Marie Curie Actions of its Sixth Program under the project O-MOORE-NICE, contract number MTKICT-2006-042477. The second and third authors acknowledge support from the EU FP5 and FP6 projects CODESTAR and CHAMELEON-RF.

\section{References}

1. Meuris, P., Schoenmaker, W., Magnus, W.: Strategy for electromagnetic interconnect modeling. IEEE Transactions on Computer-Aided Design of Integrated Circuits and Systems 20, 753-762 (2001)

2. Schoenmaker, W., Meuris, P.: Electromagnetic interconnects and passives modeling: software implementation issues. IEEE Transactions on Computer-Aided Design of Integrated Circuits and Systems 21(5), 534-543 (2002)

3. Schoenmaker, W., Meuris, P.W.S., van der Kolk K.-J. van der Meijs N.: Maxwell equations on unstructured grids using finite-integration methods. proceedings of the 12th International Conference in Simulation of Semiconductor Processes and Devices (SISPAD 2007), Vienna Austria, September 2007 (2007)

4. van der Vorst, H.A.: Iterative Krylov methods for large linear systems, Cambridge Monographs on Applied and Computational Mathematics, vol. 13. Cambridge University Press, Cambridge (2003)

5. Saad, Y.: ILUT: a dual threshold incomplete $L U$ factorization. Numer. Linear Algebra Appl. 1(4), 387-402 (1994)

6. Cuthill, E., McKee, J.: Reducing the bandwidth of sparse symmetric matrices. In: Proc. 24th Nat. Conf. ACM, pp. 157-172 (1969)

7. Amestoy, P.R., Davis, T.A., Duff, I.S.: An approximate minimum degree ordering algorithm. SIAM J. Matrix Anal. Appl. 17(4), 886-905 (1996) 